\title{
3D MODELING AND CAPACITY CALCULATION SYSTEM IN TANK CALIBRATION
}

\author{
Cuilian Zhao ${ }^{1}$, Minglun Fang ${ }^{1}$, Zhongxu Tian ${ }^{1}$, Yongsui Lin ${ }^{2}$ \\ ${ }^{I}$ Cims center of shanghai University, China; Email:clzhao@mail.shu.edu.cn ${ }^{2}$ Shanghai \\ Merchant Ship Design \& Research Institute
}

\begin{abstract}
This paper introduces a new system to calculate tank capacity based on 3D $\mathrm{CAD}$ modeling and then to get capacity forms. The whole system platform and framework are introduced, and some key technologies are also described in detailed.
\end{abstract}

Key words: $\quad 3 D$ modeling, Capacity Calculation, Tank Calibration

\section{INTRODUCTION}

Tank calibration is used to calibrate a new built or a rebuilt ship for liquid cargo trades. Tanks have double kinds of functions, on the one side, itself a storage, where a liquid cargo can be stored inside; on the other side, a meter of measurement, by checking a special document for conversing the current measured height to a volume of oil. In Australia, China, United Kingdom and some other countries, these tanks should be calibrated by an organization or company certified by the national organization of testing authorities or by the local state government trade measurement department. Obviously, the accuracy of tank calibration is important. According to the calibration regulation in China, uncertainty for oil tanker should be less than plus or minus $0.3 \%$. According to OIML Recommendation ${ }^{\mathrm{I}}$ : The relative error of the volumes stated in the document issued do not exceed: plus or minus $0.5 \%$ of the stated volume for tanks of irregular shape that cannot be calibrated using the volumetric method.

In fact, there are a various tank layout, a different cross-section, and complex inside components among tankers. Generally, tanks near tanker's 
fore are irregular, but in most of rebuilt tanks and some new built ship, there are more and more irregular tanks because of the trend to get the maximum of tank capacity. The question is how to develop a common system, which is suitable for most types of ships' situation, easy to use, make a better calibration' accuracy and take less time. With the technical development in the field of Compute Aided Design (CAD), its open platform can make full use of powerful functions. CAD is strong in describing 3D structure; its advantages in free-form feature modeling, visualization and simulation are widely accepted.

So, in this paper, a new method to calculate the irregular tank capacity faster and more accurately will be introduced. The whole system framework will be described in detailed, including some key technologies: the modification of theoretical tanker model, the way to create full oil model and etc. But in our research two following factors aren't considered:

- temperature differences between the time of calibration and the practical use.

- Pressure and deformation difference between the empty tank in calibration and the full loaded tank in the practical situation.

\section{TANK CAPACITY CALCULATION SYSTEM}

The architecture of the proposed computer-aided capacity calculation system for tank calibration is developed as shown in Figure1. Generally, the calibration operation will begin from analyzing tanker's design drawing and then measuring the tanker on site. The physical measurement can be done by a classical method - a means of tape measures, or by an optical method. So there are two kinds of input information, one is the design data, the other is the measured data. Hence, there are two data flows, which is necessary to be unified into the practical model from the model based on the design data.

Main parameters include ship's name and width, cross-section type, tanks' layout type, bottom's type, deck's type and etc. Generally, in a drawing ribs' number is used to locate the position of ship's structure, which should be transformed to absolute coordinate system in order to fulfill CAD modeling.

The oil ship is classified to three different types according to its crosssection and four types according to Bulkhead layout after we analysis a lot of ship drawings. The type of cross-section of tanker: curve type, fold type and user-defined type (a special cross-section, not included in the another two types), which decides automatically modeling function's callout for tanker's hull. Modeling with Curve type need use three degree of NURBS free-form feature modeling. Modeling with Fold type use one degree's modeling. 
According to Bulkhead layout's character, some named ways for bulkhead and longitudinal bulkhead are gives.

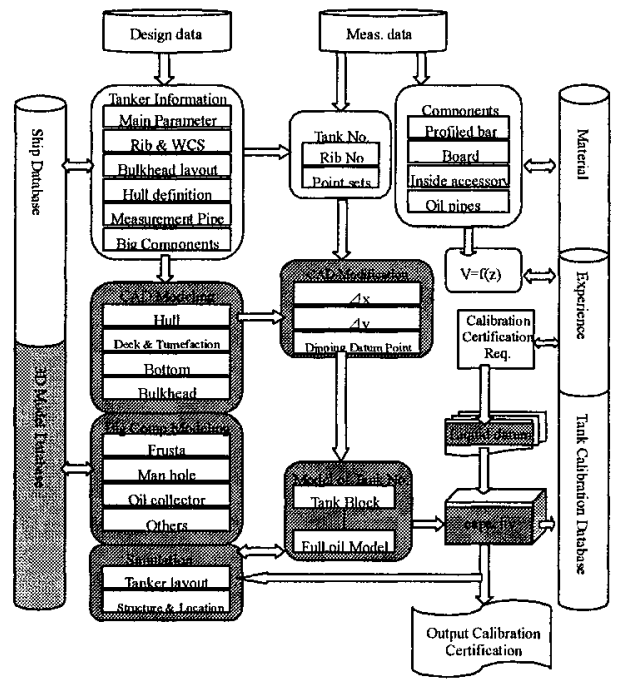

Figure 1. Architecture of 3D modeling and capacity calculation system

The above information, including 3D basic models and the expert experience, is managed by a database. We use Visual Basic 6.0 to develop the user interface, and use ACCESS 2000 as the system database, which can be accessed by a CAD open platform - UG/OPEN ${ }^{2}$ through the engine of ODBC.

Using CAD to do modeling for the hull, bulkhead, deck and some components (note: not all of components), which we consider is serious to influence the accuracy of capacity calculation. Assembly structure of a tanker will be automatically created by the developed programs. The hull and deck will be created first. With WAVE geometry linker, the hull is associated with all of other CAD components.

The output format is stipulated by the national organization of testing authorities. Automatically generate tank capacity form by the template of XLS format with the content of Ullage height, Innage height, tinl, and etc.

Figure 2 gives an example of a tank partial capacity form. Since all of data can be accessed by the tanker's database, it is easier to get a tank' conversion from a measurement height to the current capacity . 


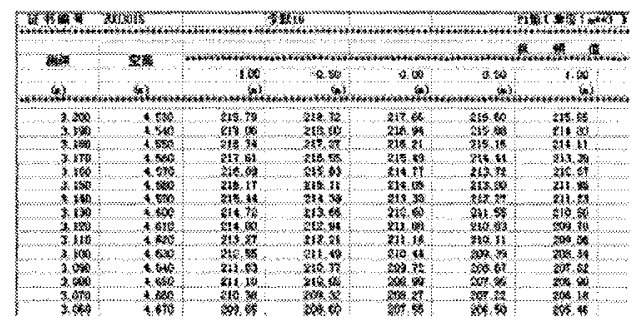

Figure 2, an example of a tank partial capacity form

\section{MODIFICATION OF TANKER MODEL}

Since all of data come from the ship design drawings, the tanker model is called theoretical model. With the developed programs, the modeling can be done automatically. in Figure 3 shows the assembly tree of a tanker and simulation of whole layout ${ }^{3}$. All the modeling based on the input data is about the shape of left side. Reference tool is used to transform the view. So it is easy to simulate the tanks' layout in a tanker, to check every component's data and to inquire the information of some key points and to know if the shape of model is right ${ }^{4}$.

However, the above model is only based on the design data. Tank Measured data is based on the tank's bottom, which is a set of points, represented by $\mathrm{Xp}, \mathrm{Yp}, \mathrm{Zpb}$. Xp is got from rib's number and offset from it, where

$\mathrm{Xp}=\mathrm{Fn}+\mathrm{N}$.

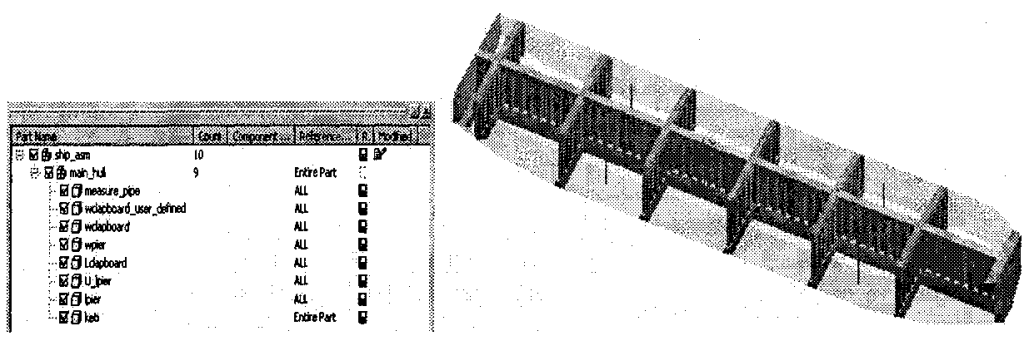

Figure 3. the assembly tree and 3D modeling of a tanker

$\mathrm{Zpb}$ is based on the bottom of tank. Firstly, need to get each tank' theoretical base point, which is the lowest point ( $\mathrm{Z}$ direction) in a tank. Then transform the measured data of each tank into absolute coordinate system, 
$Z p$. The free-form surface of hull, which has been gotten by modeling of the design data, is interpolated by the following:

$\mathrm{Y} t=\mathrm{F}(\mathrm{Xp}, \mathrm{Zp})$

Yt represents the theoretical value.

So, we can get the modification:

$$
\begin{aligned}
& \Delta \mathrm{x}=\Sigma(\mathrm{Yp}-\mathrm{Yt}) / \mathrm{n} \\
& \Delta \mathrm{y}=\Sigma(\mathrm{Xp}-\mathrm{Xt}) / \mathrm{m}
\end{aligned}
$$

where, $\mathrm{m}, \mathrm{n}$ represent the number of measured points. Similarly, there is the third important datum point in each tank - Dipping Datum Point, which decides the liquid alltitute along the vertical measurement axis. Interpolate the ship hull with each location of measurement pipe:

$\mathrm{Zj}=\mathrm{F}(\mathrm{Xj}, \mathrm{Yj})$

where, $\mathrm{Xj}, \mathrm{Yj}$ represents a measurement pipe location in a tank.

\section{FULL OIL MODEL AND TANK CAPACITY CALCULATION}

The full oil model of a tank means the oil full of the space enveloped by six sides which are free-form features or regular form features. Also the volumes of inside big components are deducted from the full oil model, such like a man hole, strong crossbeam, and etc. We made modeling of a block big enough. The six sides will cut the block which like a rough material being manufactured. Figure 4 shows the method. And then the big components will be as tools to cut the rough material continually. Using this method avoids the strict requirement for calculating a volume enveloped by sheets from CAD software. Finally the result is the full oil model in the tank.

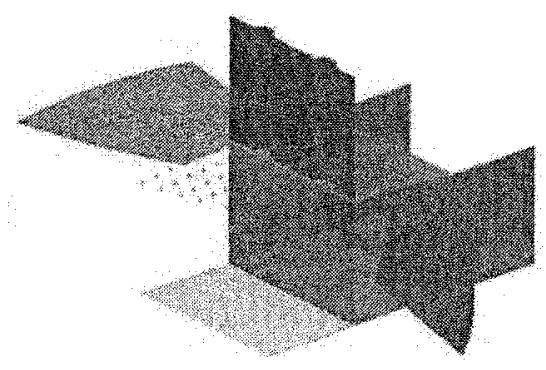

Figure 4. using a block to cut rough material using

Simulating the model, the specialist can check each tank's 3D model again. Thus, the mistake can be avoided in advance. 
After each of 3D model with full oil is ready, a liquid plane, which pass the vertical point offset from the datum point, will be created to cut off the full oil model, the left is a new model in the current measurement height and tilt. The tilt represents the difference between the height of bow and stern. The new model volume is generated by CAD volume calculation function.

For example, according to the requirement of calibration certification, the interval of heights is $10 \mathrm{~mm}$, and the interval of tilts is $0.5 \mathrm{~m}$. Hence, there will be $(6000 / 10) * 12=7200$ times of volume calculation only for a tank with $6 \mathrm{M}$ high, this is tough thing by hand. But easier to use computerizable programs.

\section{CERTIFICATE THE ACCURACY OF A TANK CAPACITY CALCULATION BASED ON CAD MODEL}

According to UG document, for simple solids with analytic faces (i.e., planar, cylindrical), the default accuracy value is sufficient. For more complicated solids with B-Surface faces, accuracy values closer to 1.0 may be necessary. The maximum accuracy value used by the system is 0.999999 (UG ref). As calibration requirement the accuracy should reach less than $0.3 \%$, according to the above, its accuracy seems to be satisfied. However, it should be certified. Since no code of CAD system is openly released. We need to prove the result of using $3 \mathrm{D}$ model calculation by mathematical theory.

Table 1. capacity results based CAD model and mathematical model

\begin{tabular}{cllll}
\hline $\begin{array}{c}\text { Altitude } \\
(\mathrm{mm})\end{array}$ & $\begin{array}{c}\text { UG } \\
\text { Volume }\end{array}$ & $\begin{array}{l}\text { Math } \\
\text { Result }\end{array}$ & $\begin{array}{l}\text { Absolute } \\
\text { Error }\end{array}$ & $\begin{array}{l}\text { Relative } \\
\text { Error }\end{array}$ \\
\hline 268 & 0.5323803 & 0.532639 & 0.000259 & 0.0005 \\
735 & 1.561296 & 1.560722 & -0.00057 & 0.0004 \\
738 & 1.568156 & 1.567582 & -0.00057 & 0.0004 \\
825 & 1.768082 & 1.76752 & -0.00056 & 0.0003 \\
1337 & 3.011928 & 3.01129 & -0.00064 & 0.0002 \\
1340 & 3.019673 & 3.019045 & -0.00063 & 0.0002 \\
1341 & 3.022256 & 3.021909 & -0.00035 & 0.0001 \\
1417 & 3.220681 & 3.220838 & 0.000157 & 0.0000 \\
1420 & 3.228597 & 3.228728 & 0.000131 & 0.0000 \\
1456 & 3.323487 & 3.323766 & 0.000279 & 0.0001 \\
1459 & 3.331465 & 3.331715 & 0.00025 & 0.0001 \\
\hline
\end{tabular}


A mathematical model of the simplest tank is created to make comparison with UG result. We create mathematical model and CAD model for computing a test tank, which is a standard tank by volumetrically calibration. Using grid with $2500 \times 2500$, the result is satisfied. See the table 1. This proves the higher accuracy using CAD model to calculate volume. So tank capacity calculation can be based on CAD model.

\section{SUMMARY}

The overall of framework of tank capacity calculation has been developed and introduced in this paper. The objective of the research is to provide a computerized tool for rapid and accurate calibration of tank capacity with simulation of 3D modeling. In the system, tank information is organized by the tank layout and cross section, tanker modeling based assembly makes the simulation for whole tanker or a special tank easily. And the modification for a practical manufacturing error can be achieved through interpolating the theoretical free-form features in CAD model. Finally, the result of tank capacity can be exported into XLS according to the regular templates. This system has already been used by Shanghai Merchant Ship Design \& Research Institute. The result proves it is accuracy enough to using CAD model for tank's capacity.

\section{ACKNOWLEDGEMENTS}

This paper is partially supported by Shanghai Merchant Ship Design \& Research Institute, authors would like to thank Yonggang, Lu, Qinwen Sun, and two graduate students from Shanghai University, Hong Zhang, Hualong Yang for their contribution in the system development.

\section{REFERENCES}

1. http://www.oiml.org First Committee Draft Revision OIML International Recommendation R71 21 - 22 April 2004, Vienna, Austria

2. V18.0 Unigraphics Help

3. Zhang Hong, Zhao Cuilian, Yang Hualong, Lin Yongsui, Research on Ship Component Management Based on Model Structure Tree , Computer Aided Engineering, 2004.9

4. Zhao Cuilian, Yang Hualong, Zhang Hong, Lin Yongsui, 3D Modeling and Simulation of Cabin Based on Assembly Environment, $C A D / C A M, 2004.5$ 